\title{
EFFECT OF NUMERICAL NOISE ON BEAM EMITTANCE GROWTH IN PIC CODE*
}

\author{
Yuri K. Batygin \\ Stanford Linear Accelerator Center, Stanford University, Stanford, CA 94309, USA
}

\begin{abstract}
In particle-in-cell (PIC) simulation, errors in space charge forces are of random character. It results in an unphysical increase of effective beam emittance, even while symplectic integrator is used. To establish quantitative measure of this effect on beam dynamics, an analytical model of equilibrium beam affected by random errors in space charge field calculations is considered. An explicit expression connecting beam emittance growth with beam brightness, integration step and the value of random error in space charge field is discussed.
\end{abstract}

\section{INTRODUCTION}

In PIC simulations space charge forces of the beam are calculated via direct solution of Poisson's equation at every elementary step of simulation. Numerical solution is affected by various errors caused by discrete charge representation used in the macroparticle method, approximation formulas used in place of the exact derivatives of the Poisson's equation, differentiation of the potential function to obtain values of electric field components, and computer round-off errors. These errors act on beam dynamics as a noise which eventually transforms into unphysical emittance growth of the beam (see Fig. 1).

\section{BEAM EMITTANCE GROWTH}

Consider linear oscillator $\ddot{x}+\omega^{2} x=0$, where $\omega$ is the space-charge-depressed oscillation frequency, affected by random errors in space charge field calculations. Matrix of particle transformation in coordinates ( $x$, $\mathrm{p}=\frac{1}{\omega} \frac{\mathrm{dx}}{\mathrm{dt}}$ ) at every elementary step, $\delta \mathrm{t}$, is given by

$$
\left(\begin{array}{l}
\mathrm{x}_{\mathrm{n}+1} \\
\mathrm{p}_{\mathrm{n}+1}
\end{array}\right)=\left(\begin{array}{cc}
\cos \theta & \sin \theta \\
-\sin \theta & \cos \theta
\end{array}\right)\left(\begin{array}{c}
\mathrm{x}_{\mathrm{n}} \\
\mathrm{p}_{\mathrm{n}}+\Delta \mathrm{p}_{\mathrm{n}}
\end{array}\right)
$$

where $\theta=\omega \cdot \delta \mathrm{t}$ is a tune shift of particle oscillation per integration step and $\Delta \mathrm{p}_{\mathrm{n}}$ is a random kick due to error $\delta \mathrm{E}_{\mathrm{n}}$ in space charge field:

$$
\Delta \mathrm{p}_{\mathrm{n}}=\frac{\mathrm{q} \delta \mathrm{E}_{\mathrm{n}} \delta \mathrm{t}}{\mathrm{m} \omega}
$$

For further analysis, let us introduce a reduced value of error in space charge field:

\footnotetext{
* Work supported by the Department of Energy contract DEAC03-76SF00515
}

$$
\vartheta=\frac{\delta E(R)}{E(R)}
$$

where $E(R)$ is an exact value of space charge field at the beam boundary:

$$
\mathrm{E}(\mathrm{R})=\frac{\mathrm{I}}{2 \pi \varepsilon_{\mathrm{o}} \beta_{\mathrm{z}} \mathrm{cR}},
$$

and $\delta \mathrm{E}(\mathrm{R})$ is a deviation of space charge field from the value of $E(R)$.

Solution of matrix equation (1) after $\mathrm{n}$ steps of integration is [1]

$$
\left\{\begin{array}{c}
\mathrm{x}_{\mathrm{n}}=\mathrm{R} \cos (\mathrm{n} \theta+\Psi)+\sum_{\mathrm{i}=0}^{\mathrm{n}-1} \Delta \mathrm{p}_{\mathrm{i}} \sin (\mathrm{n}-\mathrm{i}) \theta \\
\mathrm{p}_{\mathrm{n}}=-\mathrm{R} \sin (\mathrm{n} \theta+\Psi)+\sum_{\mathrm{i}=0}^{\mathrm{n}-1} \Delta \mathrm{p}_{\mathrm{i}} \cos (\mathrm{n}-\mathrm{i}) \theta
\end{array},\right.
$$

where $\Psi$ is an initial phase of oscillations. Second-order momentum of particle distribution in phase space are

$$
\begin{aligned}
& \left\langle x_{n}^{2}\right\rangle=\frac{1}{2 \pi} \int_{-\pi}^{\pi} x_{n}^{2} d \Psi, \\
& \left\langle p_{n}^{2}\right\rangle=\frac{1}{2 \pi} \int_{-\pi}^{\pi} p_{n}^{2} d \Psi .
\end{aligned}
$$

Random kicks $\Delta \mathrm{p}_{\mathrm{i}}$ are not correlated, therefore

$$
\Delta \mathrm{p}_{\mathrm{k}} \sin (\mathrm{n}-\mathrm{k}) \theta \sum_{\mathrm{i}=0}^{\mathrm{n}-1} \Delta \mathrm{p}_{\mathrm{i}} \sin (\mathrm{n}-\mathrm{i}) \theta=\left\{\begin{array}{l}
0, \\
\Delta \mathrm{p}^{2} \sin ^{2}(\mathrm{n}-\mathrm{k}) \theta, \mathrm{k}=\mathrm{i}
\end{array}\right.
$$

where $\Delta \mathrm{p}$ is an amplitude of random kicks. After $\mathrm{n}$ steps, the rms values are

$$
\begin{aligned}
& \left\langle\mathrm{x}_{\mathrm{n}}^{2}\right\rangle=\frac{\mathrm{R}^{2}}{2}\left[1+\left(\frac{\Delta \mathrm{p}}{\mathrm{R}}\right)^{2} \mathrm{n}\right], \\
& \left\langle\mathrm{p}_{\mathrm{n}}^{2}\right\rangle=\frac{\mathrm{R}^{2}}{2}\left[1+\left(\frac{\Delta \mathrm{p}}{\mathrm{R}}\right)^{2} \mathrm{n}\right] .
\end{aligned}
$$

Therefore, beam emittance after $\mathrm{n}$ steps, $\varepsilon_{\mathrm{n}}=4 \sqrt{\left\langle\mathrm{x}_{\mathrm{n}}^{2}\right\rangle\left\langle\mathrm{p}_{\mathrm{n}}^{2}\right\rangle}$, is related to initial emittance, $\varepsilon_{\mathrm{o}}$, as

$$
\frac{\varepsilon_{\mathrm{n}}}{\varepsilon_{\mathrm{o}}}=1+\left(\frac{\Delta \mathrm{p}}{\mathrm{R}}\right)^{2} \mathrm{n} .
$$

Let us rewrite the value of kick, Eq.(2), as 
$\mathrm{I}=0$

$\mathrm{n}=0$

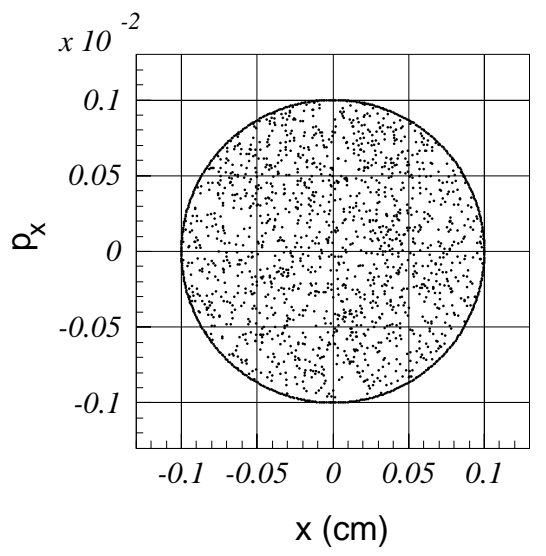

$\mathrm{n}=10^{3}$

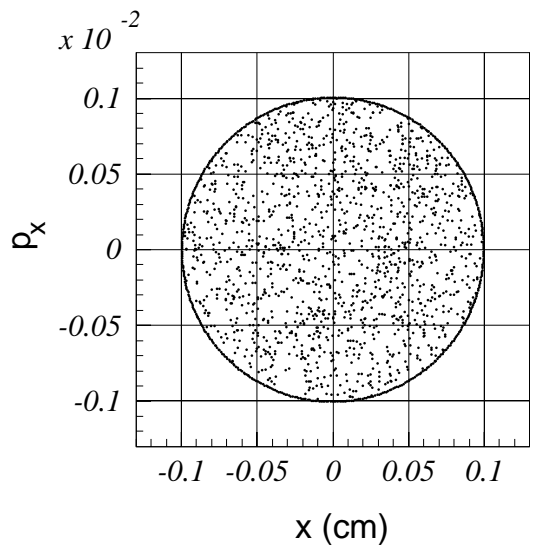

$\mathrm{I} \neq 0$
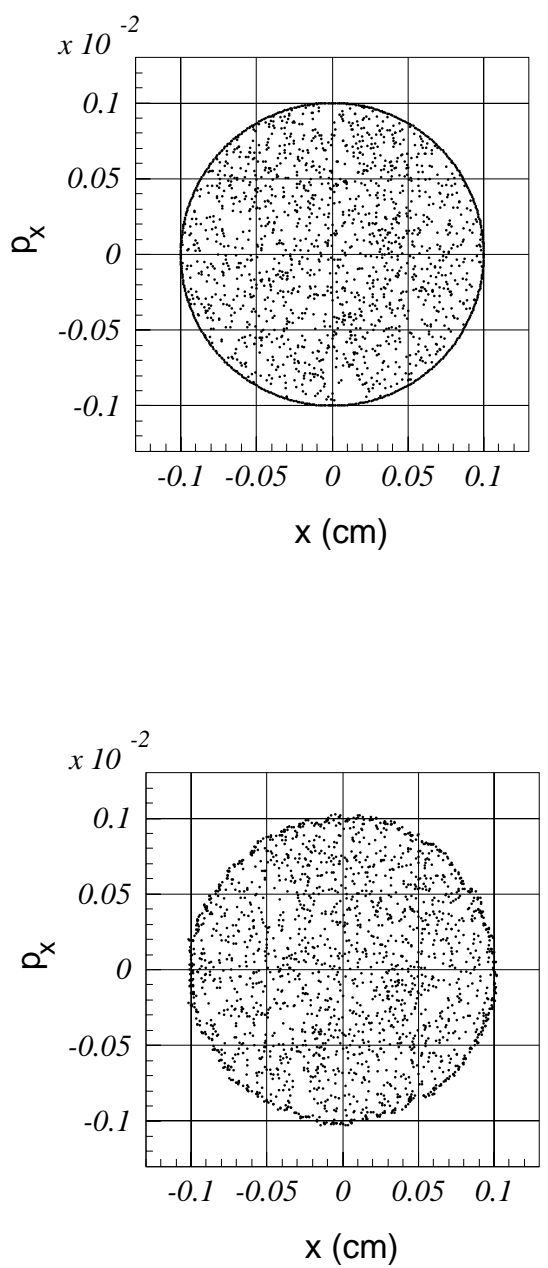

Fig. 1. Evolution of phase space area of the circular beam after $10^{3}$ integration steps with (right column) and without (left column) space charge forces.

$$
\Delta \mathrm{p}=\frac{\mathrm{q}}{\mathrm{m} \omega}\left(\frac{\delta \mathrm{E}}{\mathrm{E}}\right)\left(\frac{\delta \mathrm{t}}{\mathrm{T}}\right) \mathrm{E} \mathrm{T},
$$

where $\mathrm{T}=2 \pi / \omega$ is a period of oscillations. Also take into account that beam emittance is

$$
\ni=\frac{\mathrm{R}^{2} \omega}{\beta_{\mathrm{z}} \mathrm{c}} .
$$

Therefore, amplitude of random kick is

$$
\Delta \mathrm{p}=2 \pi \mathrm{R}\left(\frac{\delta \mathrm{E}}{\mathrm{E}}\right)\left(\frac{\delta \mathrm{t}}{\mathrm{T}}\right) \frac{2 \mathrm{I}}{\mathrm{I}_{\mathrm{c}} \beta_{\mathrm{z}}^{3}} \frac{\mathrm{R}^{2}}{\ni^{2}},
$$

where $I_{c}=4 \pi \varepsilon_{0} \mathrm{mc}^{3} / \mathrm{q}=(\mathrm{A} / \mathrm{Z}) \cdot 3.13 \cdot 10^{7} \mathrm{amp}$ is a characteristic value of the beam current.

To evaluate significance of space charge forces on beam dynamics, consider $\mathrm{KV}$ envelope equation for round beam:

$$
R^{\prime \prime}-\frac{\partial^{2}}{R^{3}}+k(z) R-\frac{2 I}{I_{c} \beta^{3} \gamma^{3} R}=0
$$

Equation (15) contains two defocusing terms: one is proportional to square of beam emittance and another one is proportional to beam current. Ratio of that two terms gives estimation, which factor dominates in beam transport:

$$
\mathrm{b}=\frac{2}{(\beta \gamma)^{3}} \frac{\mathrm{I}}{\mathrm{I}_{\mathrm{c}}} \frac{\mathrm{R}^{2}}{\ni^{2}}
$$

Parameter b, Eq. (16), is a ratio of beam brightness, I / $\ni^{2}$, to normalization value, $I_{c} / R^{2}$, and can be called dimensionless beam brightness. Space charge dominated beam transport is performed when $b>>1$ while emittance dominated regime is fulfilled for $b<<1$. Additional factor of $2 /(\beta \gamma)$ indicates that significance of space charge 
forces drops with increasing of beam energy. Finally, beam emittance growth due to random variations in space charge field calculations is

$$
\frac{\varepsilon_{\mathrm{n}}}{\varepsilon_{\mathrm{o}}}=1+\left(2 \pi \mathrm{b} \tau \frac{\delta \mathrm{E}}{\mathrm{E}}\right)^{2} \mathrm{n},
$$

where $\tau=\delta \mathrm{t} / \mathrm{T}$ is the dimensionless integration step. From Eq. (17) it follows that emittance growth is the most essential for high brightness beams.

\section{NUMERICAL SIMULATIONS}

In Fig. 2 an example of beam emittance growth due to errors in space charge field calculations is presented. Calculations are performed using code BEAMPATH [2]. Integration of equations of motion are done utilizing symplectic integrator:

$$
\vec{p}_{n+1}=\vec{p}_{n}+\tau \vec{E}_{n}, \quad \vec{x}_{n+1}=\vec{x}_{n}+\tau \frac{\vec{p}_{n+1}}{\gamma_{n+1}},
$$

where electric field, $\vec{E}=\vec{E}_{f}+\vec{E}_{s c}$, is a combination of focusing field, $\overrightarrow{\mathrm{E}}_{\mathrm{f}}$, and space charge field, $\overrightarrow{\mathrm{E}}_{\mathrm{sc}}$. After 9000 integration steps emittance growth for the KV beam with $\mathrm{A} / \mathrm{Z}=1, \mathrm{I}=4.16 \mathrm{amp}, \mathrm{R}=1 \mathrm{~mm}, \beta_{\mathrm{z}}=0.015648$, $\ni=6.39 \pi \mathrm{cm} \mathrm{mrad}, \mathrm{b}=17, \delta \mathrm{E}(\mathrm{R}) / \mathrm{E}(\mathrm{R})=0.01$ is $\varepsilon_{\mathrm{n}} / \varepsilon_{\mathrm{o}}$ $=1.025$. Beam is represented by $3 \cdot 10^{4}$ particles on the grid $\mathrm{N}_{\mathrm{x}} \times \mathrm{N}_{\mathrm{y}}=512 \times 512$. Analytical estimation of beam emittance growth utilizing formula (17) gives $\varepsilon_{\mathrm{n}} / \varepsilon_{\mathrm{o}}=$ 1.1. Without space charge forces effective beam emittance is conserved (see Fig. 2b).

Eq. (17) indicates that emittance increases with the number of integration steps. Total number of steps at the integration period $\left[0, \tau_{\text {fin }}\right]$ is

$$
\mathrm{n}=\frac{\tau_{\text {fin }}}{\tau} .
$$

Substitution of Eq. (19) into Eq. (17) gives:

$$
\frac{\varepsilon_{\mathrm{n}}}{\varepsilon_{\mathrm{o}}}=1+\left(2 \pi \mathrm{b} \frac{\delta \mathrm{E}}{\mathrm{E}}\right)^{2} \tau_{\text {fin }} \tau .
$$

According to Eq. (20), emittance growth at the fixed distance of integration is depressed for smaller values of integration step, $\tau$. Fig. 3 contains numerical results of beam dynamics with different values of integration step at the fixed integration period.

Let us note that presented rms beam emittance growth does not violate Liouville's theorem, because it is growth of the effective phase space area occupied by the beam. Both numerical integrator, Eq. (18) and matrix, Eq.(1), are symplectic, i.e. conserving microscopic phase space of the beam. Effective beam emittance can increase while microscopic emittance is conserved (see for example, Ref. [3], Fig. 4.8, page 198). Fig. 1 illustrates evolution of phase

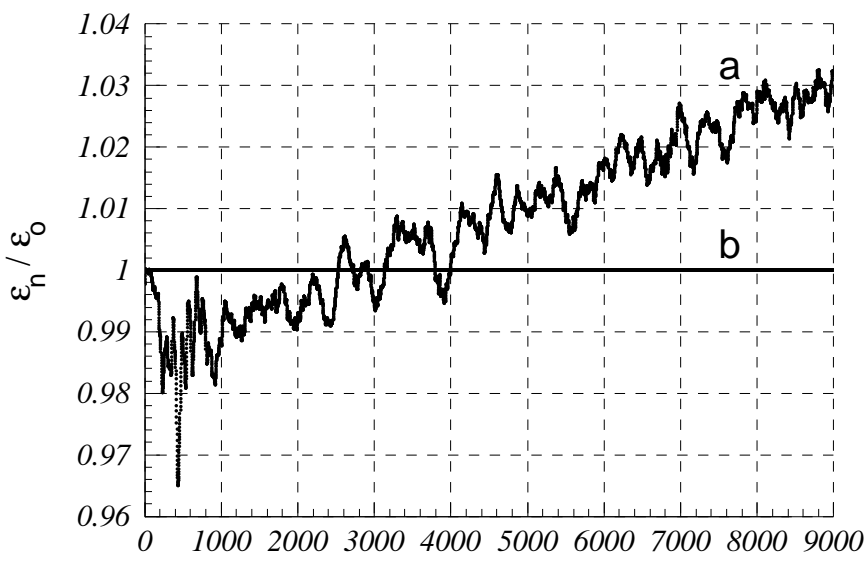

Fig. 2. Emittance growth of circular beam, $\tau=1 / 300:$ a) $b=17, \delta E(R) / E(R)=0.01, b) b=$ 0 .

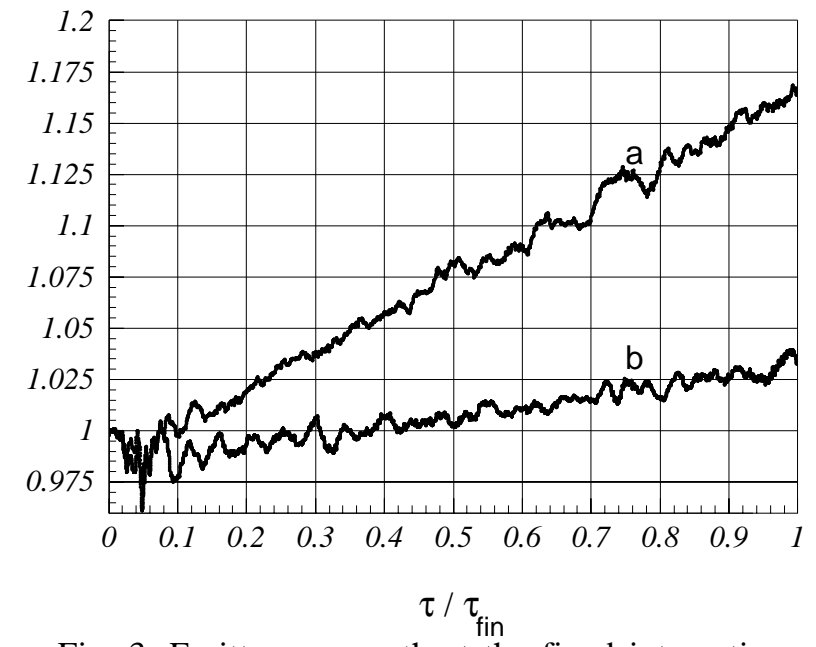

Fig. 3. Emittance growth at the fixed integration period: a) $\tau=1 / 150$, b) $\tau=1 / 300$.

space area occupied by the beam. Without space charge forces beam boundary in phase space remains unchanged. With space charge forces beam boundary is distorted due to random fluctuations in space charge field, which results in increase of effective phase space area. Meanwhile, microscopic phase space area and number of particles inside the area are conserved.

\section{REFERENCES}

[1] V.Lebedev, V.Parchomchuk, V.Shiltsev and G.Stupakov, Part. Accel. 44, 147 (1994).

[2] Y.Batygin, Proceedings of EPAC92, Editors: H.Henke, H.Homeyer and Ch. Petit-Jean-Genaz, Editions Frontiers ISBN 2-86332-114-5, p. 822 (1992); also RIKEN-AF-AC-17, ISSN 1344-3877 (2000).

[3] J.D.Lawson, The Physics of Charged-Particle Beams, Clarendon Press, Oxford, 1977. 